\title{
On the drying kinetics of non-spherical particle-filled polymer films: A numerical study
}

\section{Víctor-Alfonso Gracia-Medrano-Bravo ㄴ | Philip Scharfer | Wilhelm Schabel}

Thin Film Technology (TFT), Karlsruhe Institute of Technology (KIT), Karlsruhe, Germany

\section{Correspondence}

Víctor-Alfonso Gracia-Medrano-Bravo, Thin Film Technology (TFT), Karlsruhe Institute of Technology (KIT), Karlsruhe, Germany.

Email: victor.bravo@kit.edu

\section{Funding information}

Consejo Nacional de Ciencia y Tecnología; Deutsche Forschungsgemeinschaft; Deutscher Akademischer Austauschdienst; Karlsruhe Institute of Technology

\begin{abstract}
During the coating and drying of thin polymer-particle composites, the particle geometry has a big impact on the prediction of concentration profiles in the dry film. In this work, a plate-like geometry is used to evaluate the mass transport of the particles with the aspect ratio as a variable. The experimental determination of the viscosity and sedimentation rates allows to simulate concentration profiles in the wet film while drying. A previous simulation model was automated to describe the drying of the plate-like particles-polyvinyl alcohol-water material system using COMSOL with the initial concentration, aspect ratio, Péclet number, and sedimentation number as input parameters. The results are summarized in drying regime maps, which show an increase in the evaporation regime when the aspect ratio decreases due to lower particle mobility. This shows the importance of the geometry while predicting the particle distribution in the dry film and designing coating and drying processes.
\end{abstract}

KEYWORDS

coating, drying, drying regime maps, plate-like particles, polymer-particle composites

\section{1 | INTRODUCTION}

The use of polymer-particle composites has gained the attention of the process engineering community in the last decade, because of the improvements that embedded particles can offer to different types of functionalized polymer films. Some examples include OLEDs, ${ }^{1}$ fuel cell membranes, ${ }^{2,3}$ lithium-ion batteries, ${ }^{4}$ and biosensors. ${ }^{5}$ During the production of said functionalized films, the location of the components along the film height is important to ensure the quality of the final product. Cardinal et al. ${ }^{6}$ summarize the different possibilities for spherical particles to accumulate unidimensionally in charts for easy component distribution predictions based on the Péclet $\left(P e_{C}\right)$ and sedimentation $\left(N_{S}\right)$ numbers, defined in Equation (1):

$$
P e_{C}=\frac{E_{0} \cdot h_{0}}{D_{C, 0}} ; N_{S}=\frac{U_{C, 0}}{E_{0}}
$$

Here, $E_{0}$ is the initial solvent evaporation rate, $h_{0}$ the initial film height, $D_{C, 0}$ and $U_{C, 0}$ are the diffusion coefficient and the sedimentation rate of a single particle.

During the drying process, the particles can either accumulate at the top (evaporation regime), sink to the bottom next to the substrate (sedimentation regime), or remain evenly distributed along the film height (diffusion regime). Corresponding drying regime maps are calculated using the conservation equations of the components and the borders are defined by the criterion that the particle concentration reaches $90 \%$ of the maximum packing concentration.

If the geometry of the particles is changed, three main aspects must be taken into consideration in order to properly model the mass transport during drying: the change of the maximum packing concentration, the orientation of the particles while drying, and the changing influence of the concentration dependency on the 
viscous forces acting on the particles during diffusion and/or sedimentation.

In a previous work, the influence of the geometry was studied to experimentally determine the location of the particles during drying and extrapolate the results to the dry film. It was found that flat particles align themselves with the substrate after the coating process due to shear stress, ${ }^{7,8}$ and remain horizontal during drying and sedimentation. ${ }^{9}$ This orientation increases the drag force, making the vertical diffusion and sedimentation of plate-like particles considerably slower in comparison to spherical particles with the same volume. The change of the particle geometry also affects the way the diffusion coefficients of single particles and the sedimentation rates are calculated. Both parameters are obtained by balancing the forces which make the particles move, including gravity, buoyancy, and viscous resistance or drag. ${ }^{10}$ The drag coefficient is calculated by integrating the stress exerted from the fluid onto the particle surface, which it is dependent on the particle orientation as shown by Perrin. ${ }^{11}$

The concentration dependency of sedimentation and diffusion can be modeled using expressions for the relative viscosity of polymer-solvent-systems analogous to Batchelor ${ }^{12}$ via the sedimentation coefficient $K\left(\phi_{C}\right)$. In the literature, different models can be found for the concentration dependency. In this manuscript, a general model from Russel ${ }^{13}$ $K\left(\phi_{C}\right)=\left(1-\phi_{C}\right)^{-K_{2}}$ is utilized. This model reduces to the Batchelor ${ }^{12}$ expression at small particle concentrations. The exponent $K_{2}$ is a main subject of the present work. Depending on the particle shape, this parameter changes its value and thus the mass transport properties of the particles.

An analytical expression for $K_{2}$ for spherical particles can be found in the literature. ${ }^{14}$ It is derived from the creeping flow and the Oseen ${ }^{15}$ equations as a function of the ratio between particle radius and particle center-to-center distance. The equations consider the hydrodynamic interactions and the potential between the particles, which translate to the hindrance caused by the increase of the particle concentration. A more detailed explanation regarding the modeling of $K_{2}$ can be found elsewhere. ${ }^{16,14}$ In Table 1, there is a range of experimentally obtained $K_{2}$ for different material systems using different measurement techniques. He et al. ${ }^{17}$ use this approach to obtain a similar function for ellipsoids dependent on the aspect ratio $r_{p}$ (see Figure 1) as shown in Equation (2)

$$
K_{2}\left(r_{p}\right) \approx-\frac{3.80}{r_{p}}
$$

Unfortunately, this expression does not concord with experimental values from literature using different measuring techniques (see Table 1 and Figure 1). Values for non-spherical particles with different aspect ratios show a trend of lower mobility at lower aspect ratio for plate-like particles. For this reason, the literature data were fitted using Equation (3) to easily predict the sedimentation rate of plate-like particles. Expression (3) is based on the potential between two particles by modifying the expression from Weeks et al. ${ }^{18}$ to better describe the data in Table 1, where $A$ is the minimum value given by the spheres, $B$ the rate of decrease and $C$ defines a threshold value at
TAB LE 1 Experimentally determined $K_{2}$ values from different sources for particles at different particle aspect ratios

\begin{tabular}{lllll}
$r_{p}(-)$ & $\phi_{C, \text { max }}\left(\mathrm{m}^{3} / \mathrm{m}^{3}\right)$ & References & $K_{2}(-)$ & References \\
1.00 & 0.64 & 13 & {$[-4.25,-6.6]$} & $16,20-22$ \\
0.15 & 0.61 & 19 & -5.80 & 23 \\
0.10 & 0.52 & 19 & -11.48 & 24 \\
0.08 & 0.50 & 19 & -10.90 & 23 \\
0.03 & 0.37 & 19 & -44.90 & This work \\
\hline
\end{tabular}

Note: The $\phi_{\mathrm{C} \text {,max }}$ are reported from Chaikin ${ }^{19}$ and Russel. ${ }^{13}$

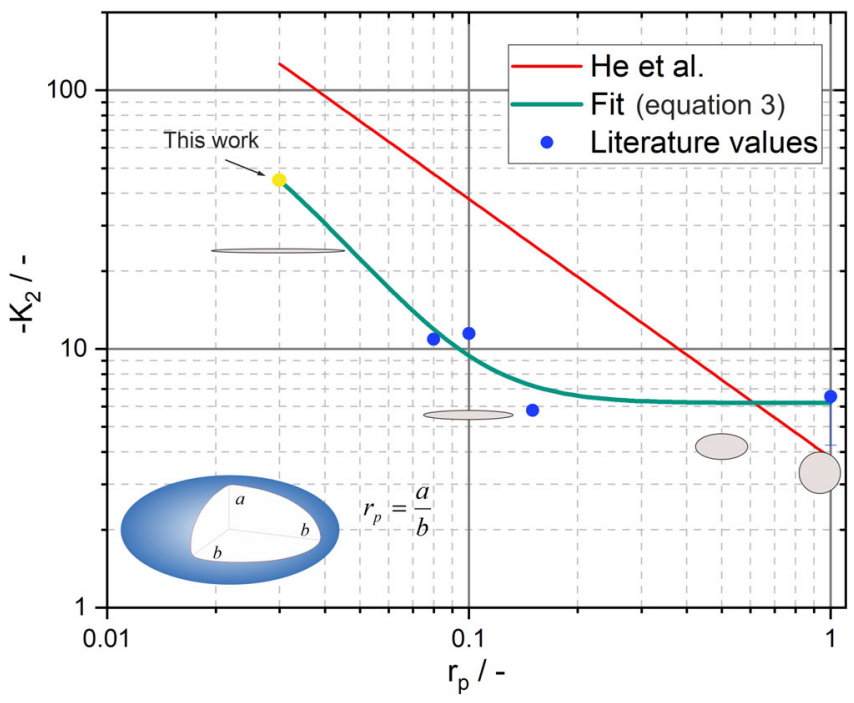

FIGURE 1 Dependence of $K_{2}$ on the particle aspect ratio. Showing the deviations between the He et al. ${ }^{17}$ model in red and literature values listed in Table 1. It can be seen that at $r_{p}=0.1$ and lower, the particle mobility decreases dramatically. A proposed model fitted from the experimental values is plotted in green with the equation $-K_{2}\left(r_{p}\right)=A+B \cdot\left[\left(r_{p}+C\right)^{-4}-\left(r_{p}+C\right)^{-2}\right]$ and the parameters $A=6.18, B=1.78 \cdot 10^{-3}, C=5.22 \cdot 10^{-2}$. Some particles with $r_{p}$ at $1,0.5,0.1$, and 0.03 are inserted to illustrate how the particles look like at different aspect ratios using the same volume

which $K_{2}$ decreases rapidly. For a better understanding of the particle aspect ratio influence on the sedimentation rate, some exemplary particle geometries with the same volume are added as sketches to Figure 1.

$$
\begin{aligned}
& -K_{2}\left(r_{p}\right)=A+B \cdot\left[\frac{1}{\left(r_{p}+C\right)^{4}}-\frac{1}{\left(r_{p}+C\right)^{2}}\right] \\
& A=6.18, B=1.78 \cdot 10^{-3}, C=5.22 \cdot 10^{-2}
\end{aligned}
$$

For plate-like particles, the maximum packing concentration $\phi_{C \text {, max }}$ depends on the aspect ratio and also on how the particles stack themselves during coating and drying. As shown by Chaikin et al., ${ }^{19}$ if the plates are wide and thin, the maximum packing concentration will 
be small and therefore it would be easier to reach the desired concentration at lower Péclet numbers. This also affects the drying regime map borders, because the discriminant of the regime zones is dependent on $\phi_{C, \text { max }}$ (see Figure 4 and Equation (26)).

The aim of this work is to build on the previous simulation with quasi-binary models and expand it for different geometries, thus creating new regime maps which allow the formulation of coating suspension, the sizing of industrial dryers, and the selection of the drying conditions. The automated plotting of the regime maps is done utilizing a simple trial and error numerical method with small convergence times. The code utilized is provided in the supporting information. The dependence of the drying regime borders on the aspect ratio of the particles is investigated, and it is discussed how the particle mobility affects the particle accumulation and the critical values of the dimensionless numbers. The aspect-ratio-dependent $K_{2}$ exponent is calculated using the approach of Equation (3) to facilitate the automatization. Finally, the influence of the aspect ratio on the drying regime maps is compared with the influence of the initial concentration of the components.

\section{2 | MODELING}

The simulation of the composite begins after the coating process, where all the particles have aligned themselves in a horizontal position, as shown in Figure 2. There, the solvent evaporates only at the top, the particles can sediment or diffuse like the polymer, and at the bottom of the film, the coating dispersion cannot get through the substrate. Using the unidirectional quasi-binary approach of Baesch et al. as framework, ${ }^{25}$ the conservation equation for the particles considers the diffusion and sedimentation contributions, whereas the polymer and solvent equations consider just diffusion. The interactions between polymer and particle and solvent and particle are treated jointly as interactions between polymer-solvent-solution and particle. The equations are then written with the volume fractions of the three components $\phi_{C}, \phi_{P}$, and $\phi_{S}$ as the variables to be solved. The subscript $C$ is used for the particles (colloid), whereas $P$ and $S$ for polymer and solvent, respectively. The concentration profiles are therefore a function of space $x$ and time $t$. The selected material system to simulate is silica flake particles dispersed in polyvinyl alcohol (PVA) and water. Particle-size distribution and stratification are not considered in this work.

The volume flux of the particles $\Gamma_{C}$ is composed of the diffusion $D_{C}$ and sedimentation velocity $U_{C}$, as shown in Equation (4):

$$
\Gamma_{C}=-\left[D_{C}\left(\phi_{C}\right) \cdot \frac{\partial \phi_{C}}{\partial x}+U_{C}\left(\phi_{C}\right) \cdot \phi_{C}\right]
$$

The concentration-dependent particle diffusion coefficient $D_{C}\left(\phi_{C}\right)$ in Equation (4) is modeled as the product of the sedimentation coefficient $K\left(\phi_{C}\right)$, the derivative of the compressibility factor ${ }^{26} Z\left(\phi_{C}\right)$ with respect to the concentration times the concentration with a maximum packing concentration of $\phi_{C, \max }$, and the temperaturedependent diffusion coefficient of a single particle $D_{C, 0}$ with a drag coefficient $\xi_{\text {i }}$.

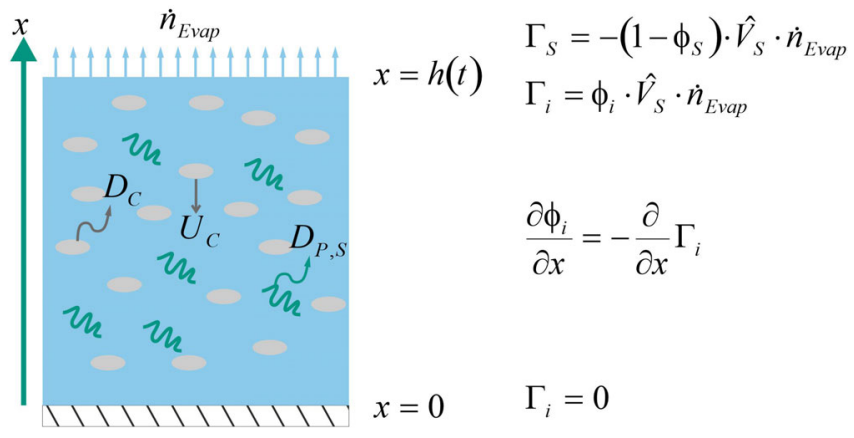

FIGURE 2 Graphical representation of the drying of polymerparticle composites. At the top of the film the solvent evaporates; the particles in the bulk sediment and diffuse, the polymer also diffuses along the film and at the bottom of the film the substrate is considered impermeable

$$
\begin{gathered}
D_{C}\left(\phi_{C}\right)=D_{C, 0} \cdot \frac{d\left[Z\left(\phi_{C}\right) \cdot \phi_{C}\right]}{d \phi_{C}} \cdot K\left(\phi_{C}\right) \\
Z\left(\phi_{C}\right)=\frac{1}{\phi_{c, \max }-\phi_{C}} D_{C, 0}=\frac{\kappa T}{\xi_{i}} .
\end{gathered}
$$

The sedimentation velocity of the particles is modeled similarly, using the sedimentation coefficient and the settling velocity of a single particle $U_{C, 0}$ with the density difference between the particles and the fluid, the gravity acceleration $g$ and the particles displaced volume $V_{C}=\frac{4}{3} \pi r_{p} b^{3}$, as shown in Equation (6).

$$
\begin{aligned}
& U_{C}\left(\phi_{C}\right)=U_{C, 0} \cdot K\left(\phi_{C}\right) \\
& U_{C, 0}=\frac{\left(\rho_{C}-\rho_{P, S}\right) g V_{C}}{\xi_{i}} .
\end{aligned}
$$

The change of the geometry is acknowledged by modifying the coefficients for diffusion/sedimentation and drag. For plate-like particles, $\xi_{i}$ is defined by Perrin's correlations, ${ }^{11}$ which are dependent on the fluid viscosity, the aspect ratio of the particles and their orientation (either parallel or orthogonal to the transport direction). As previously mentioned, the particles are aligned with the substrate after the coating process and barely change their orientation during drying. The different values used for the exponent $K_{2}$ were obtained using the fit of Equation (3). An example of the experimental determination of $K_{2}$ is displayed in Figure 3, where the concentration dependence of the sedimentation rates is fitted using Equation (6).

$$
\begin{gathered}
K\left(\phi_{C}\right)=\left(1-\phi_{C}\right)^{-K_{2}} \\
\xi=16 \cdot \pi \cdot \eta_{S} \frac{a^{2}-b^{2}}{\left(2 \cdot a^{2}-b^{2}\right) \cdot S_{i}-2 \cdot a} \\
S_{\text {oblate }}=\frac{2}{\sqrt{b^{2}-a^{2}}} \arctan \left(\frac{\sqrt{b^{2}-a^{2}}}{a}\right) .
\end{gathered}
$$



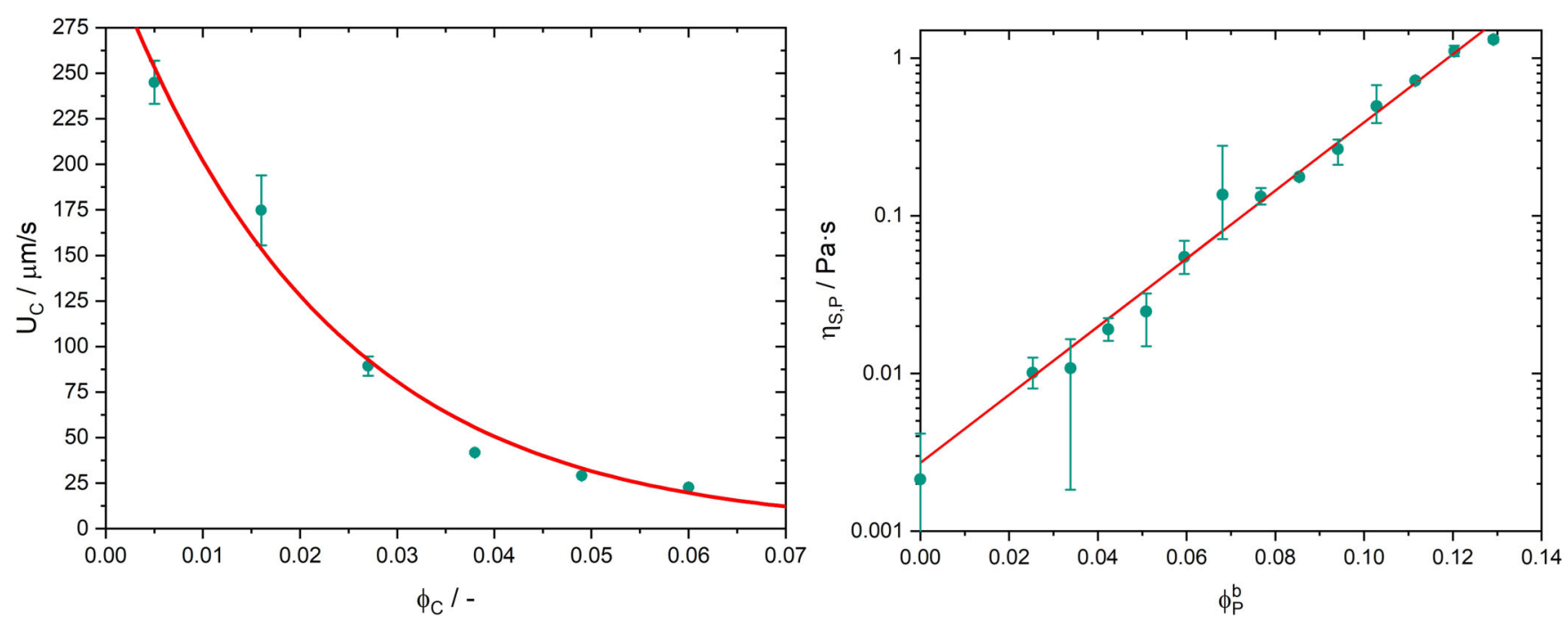

FIGURE 3 Left diagram: Sedimentation of $30 \mu \mathrm{m}$ glass flakes with an aspect ratio of $r_{p}=0.03$ in a $3 \% \mathrm{v} / \mathrm{v}$ polyvinyl alcohol (PVA) aqueous solution at different volume fractions. The experimental values are fitted using $U_{C}=U_{C, 0} \cdot\left(1-\phi_{C}\right)^{-K_{2}}$ with $U_{C, 0}=317 \mu \mathrm{m} / \mathrm{s}$ at an acceleration of $12 \mathrm{~g}$ and $K_{2}=-44.90$. Right diagram: Zero shear viscosity of polyvinyl alcohol at different concentrations and exponential fit function $\eta_{S P}=$ $A \cdot \exp \left(B \cdot \phi_{P}^{b}\right)$ with the parameters $A=2.38 \cdot 10^{-3} \mathrm{~Pa} \cdot \mathrm{s} ; B=50.9$

The polymer and solvent volume fluxes are calculated using the quasi-binary approach relative to the particle movement, using the following set of equations:

$$
\Gamma_{i}=-\left(\frac{D_{P, S}\left(X_{S}^{b}\right)}{1-\phi_{C}} \cdot \frac{\partial \phi_{i}}{\partial x}+\frac{\phi_{i}}{\left(1-\phi_{C}\right)^{2}} \cdot \frac{\partial \phi_{C}}{\partial x}\right)-\phi_{i} \cdot \Gamma_{C}
$$

where

$$
i=S, P \text {. }
$$

The diffusion coefficient of the solvent in the polymer is modeled using expression (9) according to Jeck et al. ${ }^{27}$ The parameters $A, B$, and $C$ are fitted experimentally, the values for the PVAwater system are provided below the expression. The expression has been proven to be useful in the past for describing the concentration profiles of polymer composites at other polymer molecular weight. $^{25}$

$$
\begin{aligned}
& D_{P, S}\left(X_{S}^{b}\right)=\exp -\left(\frac{A+B \cdot X_{S}^{b}}{1+C \cdot X_{S}^{b}}\right), \\
& A=37.47 ; B=321.7 ; C=14.51 .
\end{aligned}
$$

The conservation equation system is presented in Equation (10), with the Robin boundary conditions (11) to (13), where at the substrate there is no flux and at the phase boundary the solvent is evaporating. The mole flux $\dot{n}_{S}^{\text {evap }}$ can be converted to volume flux by multiplying it by the solvents molar volume $\widehat{V}_{s}$. The corresponding expressions are obtained from the mass balance at the film surface.

$$
\begin{gathered}
\frac{\partial \phi_{i}}{\partial t}=-\frac{\partial}{\partial x} \Gamma_{i}, \\
x=0 \forall t>0 \quad \Gamma_{i}=0, \\
x=h(t) \forall t>0 \quad \Gamma_{S}=-\left(1-\phi_{S}\right) \cdot \widehat{V}_{S} \cdot \dot{n}_{S}^{\text {evap }}, \\
x=h(t) \forall t>0 ; \text { for } i=C, P \quad \Gamma_{i}=\phi_{i} \cdot \widehat{V}_{S} \cdot \dot{n}_{S}^{\text {evap }} .
\end{gathered}
$$

The evaporation of the solvent is modeled with a viscous boundary layer at the phase boundary using the integrated expression (14) for $\dot{n}_{S}^{\text {evap } 28 \text { : }}$

$$
\dot{n}_{S}^{\text {evap }}=\widetilde{\rho}_{\text {gas }} \cdot \beta_{S, \text { air }} \cdot \ln \left(\frac{1-\widetilde{y}_{S}^{\infty}}{1-\widetilde{y}_{S}^{*}\left(T, \phi_{P}^{b}\right)}\right),
$$

where $\beta_{S \text {,air }}$ is the mass-transfer coefficient from the phase boundary to the top of the gas phase mass transport boundary layer. Depending on the drying process, this coefficient can be calculated from Nusselt/Sherwood number correlations found in the literature. ${ }^{29} \widetilde{\rho}_{\text {gas }}$ is the molar density of the gas phase, $\widetilde{y}_{S}^{*}\left(T, \phi_{P}^{b}\right)$ is the temperature-dependent and concentration-dependent molar fraction of the solvent at the phase boundary, it is considered to be the same concentration as in thermodynamic equilibrium, and $\widetilde{y}_{S}^{\infty}$ is the molar fraction of solvent in the air. In this work, it is assumed that the air is completely dry $\left(\widetilde{y}_{S}^{\infty}=0\right)$, which is the air condition in our laboratory experiments.

The concentration at the phase boundary is modeled according to Raoult's law $\tilde{y}_{S}^{*}\left(T, \phi_{P}^{b}\right)=a_{S}\left(\phi_{P}^{b}\right) \cdot p_{S}^{*}(T) / p_{\text {Tot }}$ with the solvent activity from the Flory-Huggins theory. The concentration-dependence of the 
binary interaction parameter $\chi_{P, S}$ is obtained from also from Jeck et al. ${ }^{27}$

$$
\begin{aligned}
& \ln a_{S}=\left(1-\phi_{P}\right)+\phi_{P}+\chi_{P, S} \cdot \phi_{P}^{2} . \\
& \chi_{P, S}=5.9340-5.4556 \cdot \phi_{P}^{0.0725} .
\end{aligned}
$$

The film thickness is obtained from the mass balance at the phase boundary. It is supposed that the drying occurs only at the top of the film and the film shrinkage is one dimensional.

$$
\frac{d h}{d t}=-E=-\widehat{V}_{S} \cdot \dot{n}_{S}^{\text {evap }} .
$$

The non-dimensionalization of the system (10) is done with the characteristic length $h_{0}$ and the characteristic time $t_{\text {Char }}=h_{0} / E_{0}$. The initial evaporation rate is calculated using expression (17).

$$
E_{0}=\widetilde{\rho}_{\text {gas }} \cdot \widehat{V}_{S} \cdot \beta_{S, \text { air }} \cdot \ln \left(\frac{1-\widetilde{y}_{S}^{\infty}}{1-\widetilde{y}_{S}^{*}\left(T, \phi_{P}^{b}\right)}\right)
$$

This leads to the inclusion of the Péclet and sedimentation numbers into the equation system and thus the dimensionless equation systems (18) and (19). The transient change of the drag coefficient due to the increasing viscosity during drying is acknowledged by the binary relative viscosity $\eta_{\text {rel }}\left(\phi_{P}^{b}\right)$ based on the initial polymer concentration.

$$
\frac{\partial \phi_{C}}{\partial \tau}=\frac{\partial \bar{\Gamma}_{C}}{\partial \bar{x}}=\frac{\partial}{\partial \bar{x}}\left[\frac{K\left(\phi_{C}\right)}{P e_{C} \cdot \eta_{\mathrm{rel}}\left(\phi_{P}\right)} \cdot \frac{d\left[Z\left(\phi_{C}\right) \cdot \phi_{C}\right]}{d \phi_{C}} \cdot \frac{\partial \phi_{C}}{\partial \bar{x}}+\frac{N_{S} \cdot K\left(\phi_{C}\right)}{\eta_{\mathrm{rel}}\left(\phi_{P}\right)} \phi_{C}\right]
$$

for $i=S, P$

$$
\frac{\partial \phi_{i}}{\partial \tau}=\frac{\partial}{\partial \bar{x}}\left[\frac{d_{P}\left(\phi_{S}\right)}{P e_{P}} \cdot\left(\frac{1}{1-\phi_{C}} \cdot \frac{\partial \phi_{i}}{\partial \bar{x}}+\frac{\phi_{i}}{\left(1-\phi_{C}\right)^{2}} \cdot \frac{\partial \phi_{C}}{\partial \bar{x}}\right)+\phi_{i} \cdot \bar{\Gamma}_{C}\right]
$$

The concentration-dependence of the zero-shear viscosity of the aqueous PVA was experimentally determined and the data were fitted using the following model. The measured viscosity is plotted in Figure 3.

$$
\begin{gathered}
\forall \phi_{P}^{b} \in[0.00,0.129]: \eta_{S, P}=A \cdot \exp \left(B \cdot \phi_{P}^{b}\right), \\
A=2.38 \cdot 10^{-3} \mathrm{~Pa} \cdot s ; B=50.9 .
\end{gathered}
$$

It is important to notice that the dimensionless evaporation rate $\bar{E}$ in Equation (21) does not require the mass-transfer coefficient to be calculated, making it independent of the air velocity.

$$
\frac{d \bar{h}}{d \tau}=-\frac{E}{E_{0}}=-\bar{E}=-\frac{\ln \left(\frac{1-\widetilde{y}_{s}^{\infty}}{1-\widetilde{y}_{S}\left(T, \phi_{P}^{b}\right)}\right)}{\ln \left(\frac{1-\widetilde{y}_{s}^{\infty}}{1-\widetilde{y}_{S, 0}\left(T, \phi_{P}^{b}\right)}\right)} .
$$

The dimensionless boundary conditions are as it follows:

$$
\bar{x}=0 \forall \tau>0 \quad \bar{\Gamma}_{i}=0,
$$

$$
\bar{x}=\bar{h}(\tau) \forall \tau>0 \quad \bar{\Gamma}_{S}=-\frac{\left(1-\phi_{S}\right) \cdot \widehat{V}_{S} \cdot \dot{n}_{S}^{\text {evap }}}{E_{0}},
$$

$$
\bar{x}=\bar{h}(\tau) \forall \tau>0 \text {; for } i=C, P \quad \bar{\Gamma}_{i}=\frac{\phi_{i} \cdot \widehat{V}_{S}^{\cdot} \cdot n_{S}^{\text {evap }}}{E_{0}} .
$$

The simulation is carried out with the initial concentration, temperature, aspect ratio, $P e_{C}$ and $N_{S}$ as input parameters. The Péclet number for the polymer $P e_{P}$ is obtained from the $P e_{C}$ and the ratio of the diffusion coefficient of solvent in polymer and the diffusion coefficient of particles in the polymer-solvent solution.

$$
P e_{P}=P e_{C} \cdot\left(\frac{D_{C, 0}}{D_{S, P, 0}}\right)
$$

\section{3 | METHODOLOGY}

\subsection{Numerical simulation}

Similarly to a previous work, ${ }^{24}$ the PDE system was solved using the commercially available software COMSOL Multiphysics 5.5 and the Application Builder with the partial differential equation general form package and a moving mesh. The regime boundaries are determined by calculating the transient particle concentration profiles at a fixed $N_{S}$ and varying the $P e_{C}$. If the particle concentration has reached a value of $90 \%$ of the maximum packing concentration at half of the time $\tau_{\max } / 2$ either at the bottom or the top, the values for the dimensionless numbers are saved and the method starts over with a new value of $N_{S}$. It must be noted that Equation (26) differs from previous publications where $\tau_{\max }$ was defined as the time needed to reach the polymer overlapping concentration. Nevertheless, the simulation has a constrain in case the polymer concentration reaches said value. If not, a new value for the $P e_{C}$ is calculated using the secant method. Aside from the start values for the numerical method, the input parameters given for the simulation are temperature $T$, concentration $\phi_{i, 0}$ and aspect ratio $r_{p}$. The numerical method is summarized in the flow chart in Figure 4. The code utilized is given in the supporting information.

$$
\tau_{\max }=\frac{\phi_{C, \max }-\phi_{C, 0}}{\phi_{C, \max }}
$$

\section{2 | Materials}

Aqueous polymer solutions were prepared by dissolving PVA $(99 \%$ hydrolyzed, Sigma-Aldrich) at a molecular weight of $\widetilde{M}_{\mathrm{PVA}}=$ $89,000-98,000 \mathrm{~g} / \mathrm{mol}$ in distilled water (Sigma-Aldrich). The particles 


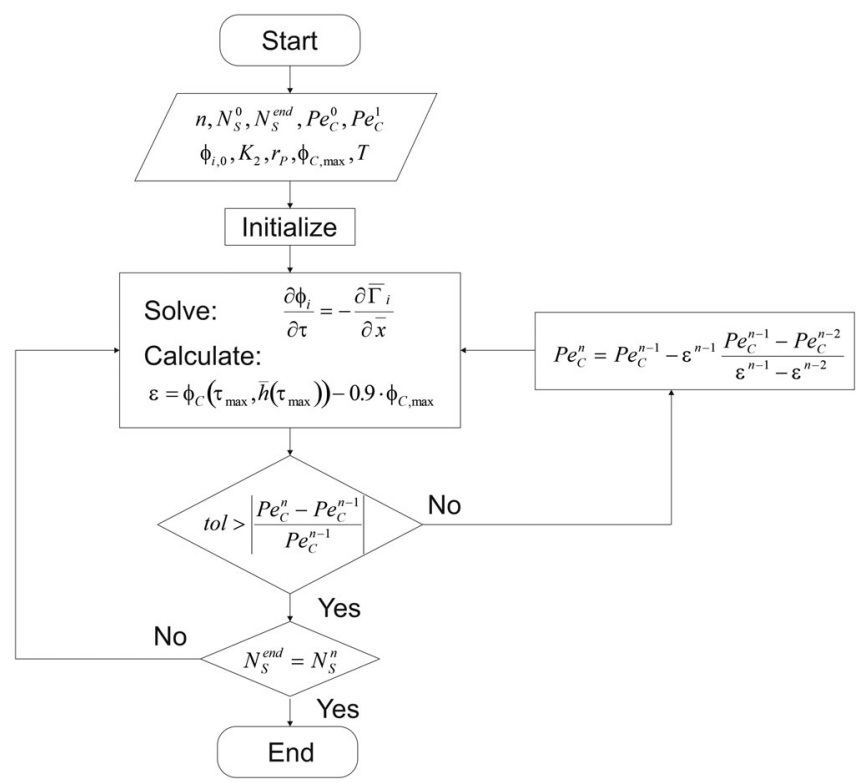

FIGURE 4 Numerical method flow chart for the evaporationdiffusion regime border. The input parameters for the program are: the number of points $n$, the interval of the drying map $\left[N_{S}^{0}, N_{S}^{\text {end }}\right]$, the initial estimation points $\left[P e_{C}^{0}, P e_{C}^{1}\right]$, initial concentration of each component $\phi_{i, 0}$, the particle shape $r_{p}$, maximum packing concentration $\phi_{C \text {, max }}, K_{2}$, and temperature. Using the input parameters, the program solves the partial differential equation system and verifies if the particle concentration at the top corresponds to $90 \%$ of $\phi_{C \text {, max }}$ by calculating the error $\varepsilon$. If the given $P e_{C}^{n}$ difference is less than the tolerance, then the program starts again with the next $N_{S}^{i}$ value, if not, a new value for the $P e_{C}^{n}$ is calculated using the secant method until it reaches convergence. The numerical approach does not change for the sedimentation regime border, only the error is calculated with the concentration at the bottom of the film $\phi_{C}\left(\tau_{\max }, 0\right)$

used were glass flakes (GF001, Glassflake Ltd.), composed mostly of $\mathrm{SiO}_{2}$, with an average particle size of $30 \mu \mathrm{m}$ and a thickness between 1.0 and $1.3 \mu \mathrm{m}$, resulting in an aspect ratio of $r_{p}=0.03$. The densities of the polymer and particles given by the manufacturer were $\rho_{P}=$ $1190 \mathrm{~kg} / \mathrm{m}^{3}$ and $\rho_{\mathrm{C}}=2600 \mathrm{~kg} / \mathrm{m}^{3}$, respectively.

\section{3 | Viscosity measurements}

The viscosity was measured using a 20-mm cone-plate geometry at $40^{\circ} \mathrm{C}$ in a rotational rheometer (Anton Paar Physica MCR101), utilizing aqueous PVA ( $99 \%$ hydrolyzed, Sigma-Aldrich $M_{\text {PVA }}=89-98 \mathrm{~kg} / \mathrm{mol}$ ) solutions at different concentrations from 0 to $15 \mathrm{wt} \%$.

\subsection{Sedimentation measurements}

Several samples were prepared using the method described above with different particle volume fractions from $\phi_{C}=0.01$ to 0.06 and a constant polymer volume fraction of $\phi_{P}=0.03$. The sedimentation rate was measured using a LUMiSizer centrifuge (LUM GmbH) in triplicate. The experiments were conducted at accelerations of $12 \mathrm{~g}$ and $40^{\circ} \mathrm{C}$ until all the particles had sedimented.

\section{RESULTS AND DISCUSSION}

In Figure 5, the concentration profiles are shown at different times during the simulation and at different values of $P e_{C}$ and $N_{S}$, exhibiting the sensitivity of these parameters. The initial solvent and particle volume fractions in the dry film chosen are $\phi_{S, 0}=0.95$ and $\phi_{C, \text { dry }}=0.33 \ldots$ respectively and the aspect ratio was $r_{p}=0.1$. The first row of plots shows the concentration profiles of particles, solvent, and polymer at $N_{S}=1$ and $P e_{C}=10$. Due to the fast evaporation of the solvent and the slow diffusion of the particles, the latter accumulate at the top of the film showing a notable gradient. The polymer however does not exhibit the same extreme gradient due to the high diffusion coefficients which counterbalance the high evaporation of the solvent. To achieve similar gradients a stronger drying rate is required. More information about polymer film drying can be found elsewhere. ${ }^{30-32}$

The next row shows the concentration profiles when all the driving forces are equal $\left(N_{S}=1, P e_{C}=1\right)$. During the drying, most of the concentration profiles remain without visible gradients until $\tau=0.7$. At this point in time, evaporation and sedimentation process are not dominant, the particle diffusion velocity does not allow an accumulation either at the top or the bottom of the film. The increasing viscosity of the liquid phase is the main reason why the particles accumulate at the top of the film, and a small concentration gradient can be seen at the last time step. In the last row at $N_{S}=10$ and $P e_{C}=10$, the particles sink immediately to the bottom of the film. Even though the evaporation is high, the sedimentation velocity overcomes the evaporation rate and the diffusion of the particles, thus not allowing the movement of the particles in the phase boundary direction.

It must be noted that the simulation cannot run properly using some combination of $\mathrm{Pe}_{C}$ and $\mathrm{N}_{S}$. If it were to obtain an accumulation of polymer and particles at the top of the film, high $P e_{C}$ and low $N_{S}$ numbers should be given to acquire such regime, due to the high diffusion velocity of the polymer in contrast to the particle. Unfortunately, to that extreme the program does not converge.

In Figure 6, a drying regime map at $r_{p}=0.10$ is displayed. It is drawn utilizing the method described in the previous section, with the condition that the $90 \%$ of $\phi_{C \text {,max }}$ must be reached without exceeding the overlap concentration of the polymer $\phi_{P}^{b}=0.13$. The initial volume fractions given for the resulting maps are $\phi_{S, 0}=0.95$ and $\phi_{C, \text { dry }}=0.33 \ldots$. The three different possibilities for the particles to accumulate are illustrated and marked with their respective symbol. The regions in the map are defined as follows: where the particles accumulate at the top of the film is the evaporation regime $(E)$, where the particles sink to the bottom at the substrate is the sedimentation regime $(S)$, and finally the region where the particle concentration will not exceed $90 \%$ of $\phi_{C \text {, max }}$ at any place in the film is the diffusion regime $(D)$. The main separation of borders is defined with the critical values of $P e_{C}, N_{S}$, and the Péclet sedimentation number $\mathrm{Pe}_{S}$, which can be calculated as a product of the other two 

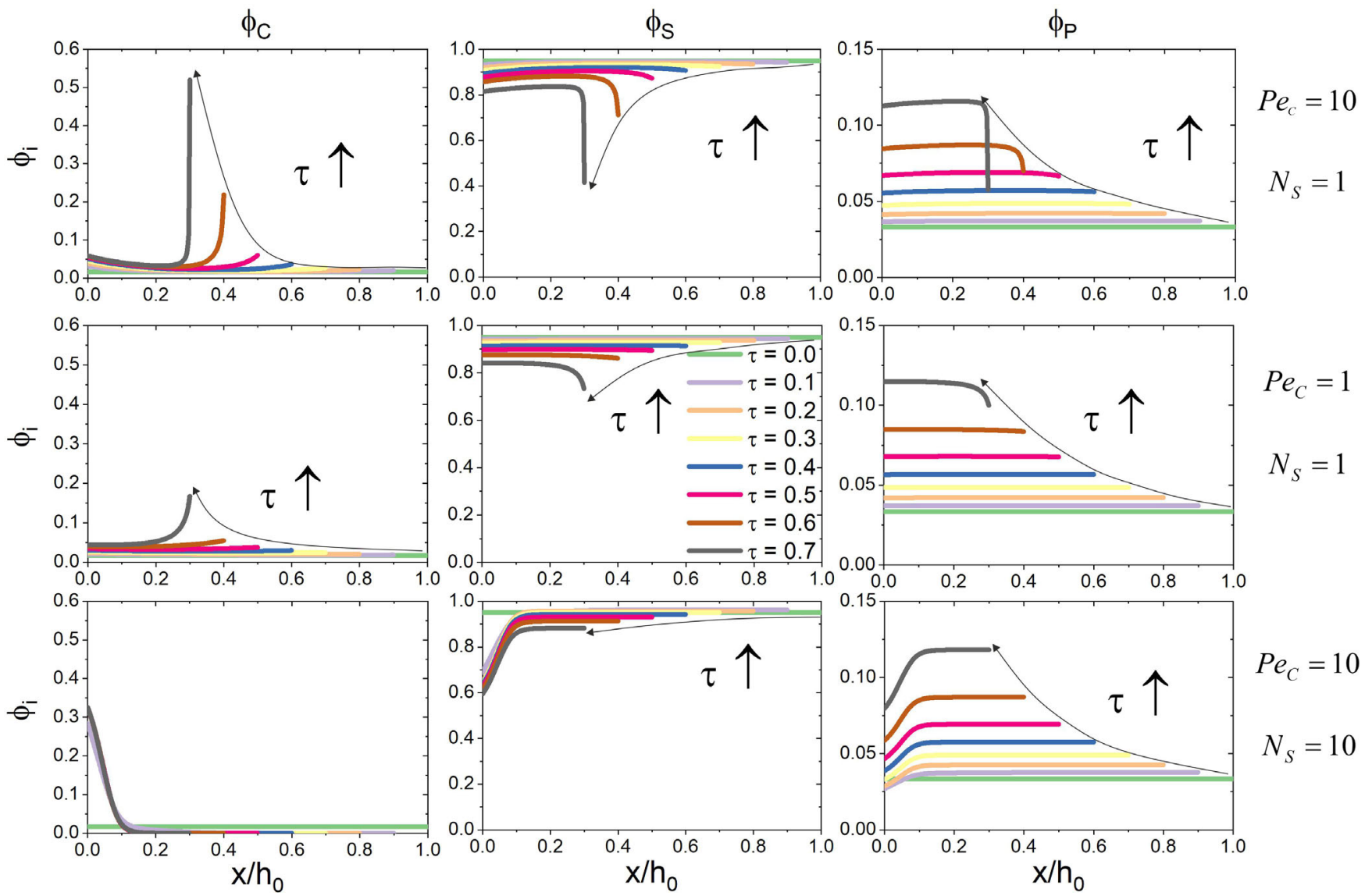

FIGURE 5 Volume fraction profiles as a function of the normalized film height at different dimensionless times, the left, middle, and right columns correspond to the concentration profiles of particles, solvent, and polymer, respectively. Calculations were done with an aspect ratio of $r_{p}=0.1$. The first row corresponds to a $P e_{C}=10$ and $N_{S}=1$ and an accumulation of the particles at the film surface can be observed. The row in the middle was calculated with $P e_{C}=1$ and $N_{S}=1$. Even at lower Pe numbers a particle accumulation at the surface is obtained due to an increase of the viscosity. The row at the bottom corresponds to $P e_{C}=10$ and $N_{S}=10$, at this high sedimentation numbers an accumulation at the bottom of the coating happens almost immediately

dimensionless numbers as shown in Equation (27). These numbers set the change when the evaporation dominates the diffusion $P e_{C, \text { crit }}$, the sedimentation dominates the evaporation $N_{S, \text { crit }}$, and the sedimentation overcomes the diffusion $P e_{S, \text { crit }}$. Graphically the $P e_{S, \text { crit }}$ can be described as the ordinate intercept of the line from the border between diffusion and sedimentation regime at high $N_{S}$ using Equation (28).

$$
\begin{gathered}
P e_{S}=\frac{U_{C, 0} \cdot h_{0}}{D_{C, 0}}=P e_{C} \cdot N_{S}, \\
\log \left(P e_{C}\right)=-\log \left(N_{S}\right)+\log \left(P e_{S, \text { crit }}\right) .
\end{gathered}
$$

The influence of the aspect ratio can be seen in Figure 7. It is immediately noticed that there is a trend for a decreasing aspect ratio, which lowers the evaporation-diffusion border, thus expanding the evaporation regime for a lower aspect ratio. The map at $r_{p}=0.03$ could not be fully calculated for the sedimentationdiffusion border with the same conditions as given for the other borders, that it is why the tolerance was lowered to achieve convergence.
The region in which the tolerance is lowered is marked with a dashed line.

The explanation of why such change of the borders occurs lies on the values of the exponent $K_{2}$, which reduces with a lower aspect ratio. A lower aspect ratio at identical particle volume results in the particles getting flatter and flatter, which would mean for the fluid around the particles a greater obstacle to overcome, and thus a greater viscous resistance to flow. And when the evaporation rate increases, the particles cannot diffuse fast enough to compensate the gradient, thus expanding the evaporation regime at a low $r_{p}$. As mentioned in the previous section, certain numerical values are not possible to simulate for the two main dimensionless numbers. The same principle applies for the sedimentation coefficient $K\left(\phi_{C}\right)$, which at $r_{p}=0.03$ is a function raised to the power of $\sim 45$.

As depicted in Figure 7 the borders follow a constant value at lower $N_{S}$ and when they approach values in the neighborhood of the $N_{S, \text { crit }}$, it starts to diverge, due to the increase in the sedimentation and diffusion driving forces. From higher $N_{S}$ until the $N_{S \text {,crit }}$ a similar behavior is observed, the border follows the line given by Equation (28) and diverge form that path when the $N_{S}$ lies under 10 , caused 


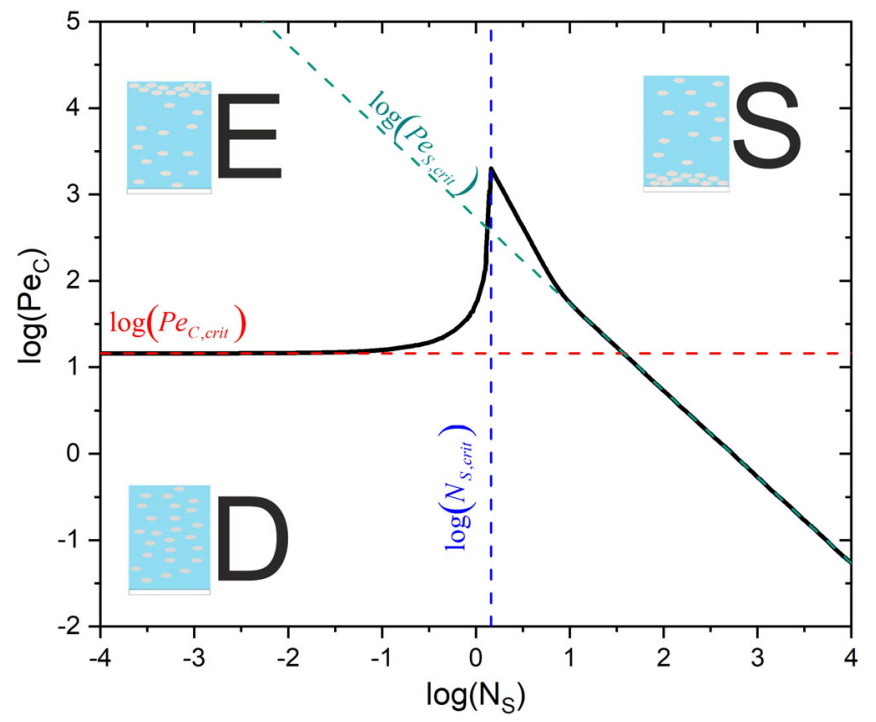

FIGURE 6 Drying regime map with initial solvent volume fraction $\phi_{S, 0}=0.95$, a particle volume fraction in the dry film $\phi_{C, \text { dry }}=0.33$, and an aspect ratio $r_{p}=0.10$. The three different regimes are illustrated and marked with the corresponding letter; evaporation regime $(E)$ : the particles accumulate at the top, sedimentation regime (S): the particles accumulate at the bottom, diffusion regime (D): the particle concentration does not reach $90 \%$ of $\phi_{C \text {, max }}$ and therefore the particles remain evenly distributed. The borders between regimes are marked in color using the critical dimensionless numbers as follows: $\mathrm{D}-\mathrm{E}$ : regime with $P e_{C, \text { crit }}$ in red, E-S: regime with $N_{S, \text { crit }}$ in blue, and S-D: regime with the line whose ordinate intercept corresponds to the $P e_{S, \text { crit }}$ in green

by increment on the diffusion. The maximum in each border is the turning point for the Evaporation and Sedimentation regimes, in which $90 \% \phi_{C, \max }$ is reached at both top and bottom of the film.

The quantification of the border shifts is shown in Figure 8, where the critical $P e_{C \text {,crit }}$ and $N_{S, \text { crit }}$ are plotted as a function of the aspect ratio. The border between evaporation and diffusion regime corresponds to the $P e_{c, \text { crit }}$, the implicit border between evaporation and sedimentation regime is quantified with the $N_{S \text {, rrit }}$, and $P e_{S, \text { crit }}$ defines the separation between the diffusion and sedimentation regime. In the studies from Cardinal et al. ${ }^{6}$ it was stated that $P e_{S, \text { crit }}$ is only dependent on the initial concentration of the particles. This is also shown by our results, since, all the borders at different $r_{p}$ overlap. The region $\forall \log \left(N_{S}\right) \in[0,1]$ shows a different trend, because sedimentation driving forces have not yet fully overcome the evaporation and the drag provoked by the flat geometry. That it is why $N_{S}$ at $r_{p}>0.03$ needs to be one order of magnitude greater in order to follow the same constant trend. However, for $r_{p}=0.03$ we cannot be certain, due to the limitation of the simulation routine mentioned above.

As it can be noticed in Figure 8, when the aspect ratio increases, the Péclet number follows an increasing trend, and on the other hand the sedimentation number decreases when particles get rounder. The trend of $P e_{C \text {,crit }}$ can be explained by the values of $K_{2}$, allowing a higher mobility as the aspect ratio of the plate-like

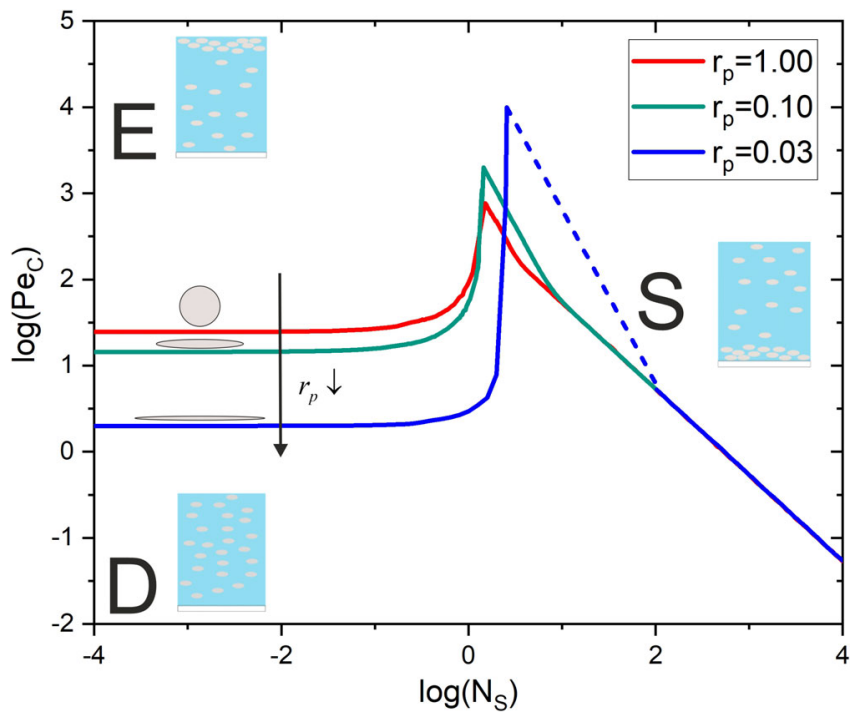

FIGURE 7 Dependence of the drying regime borders on particle aspect ratio. The lower the aspect ratio $r_{p}$, the lower the boundaries are located. The borders were simulated using the data available in the literature ${ }^{23}$ and experimentally obtained. The dashed area for an $r_{p}=0.03$ is due to the lower tolerance in the simulation to obtain convergence

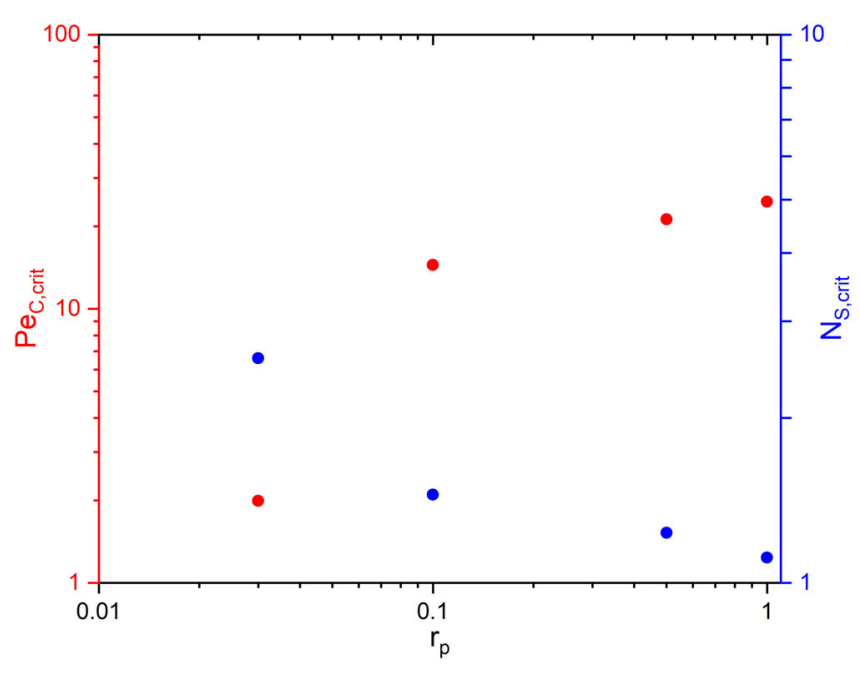

FIGURE 8 Influence of the $r_{p}$ on the critical values of the $P e_{C}$ and $N_{S}$ for plate-like particles. Showing an increase with the $P e_{C, \text { crit }}$ and a decrease with the $N_{S, \text { crit }}$ (see Figure 6)

particles increases (see Table 1). A higher mobility will imply higher diffusion coefficients, not allowing the particles to accumulate at the top of the film. Experimentally at $r_{p}=0.1$ and $r_{p}=0.08$ (see Table 1) it seems that the trend is not being followed, but those values are very close to each other and due to the different measuring techniques used to determine $K_{2}$, it should be interpreted as a small deviation from different literature sources rather than a break of the trend.

At $N_{S, \text { crit }}$, is assumed that the evaporation rate is approximately the sedimentation of the particles at $x=h\left(t_{0}\right)$. In other words, 

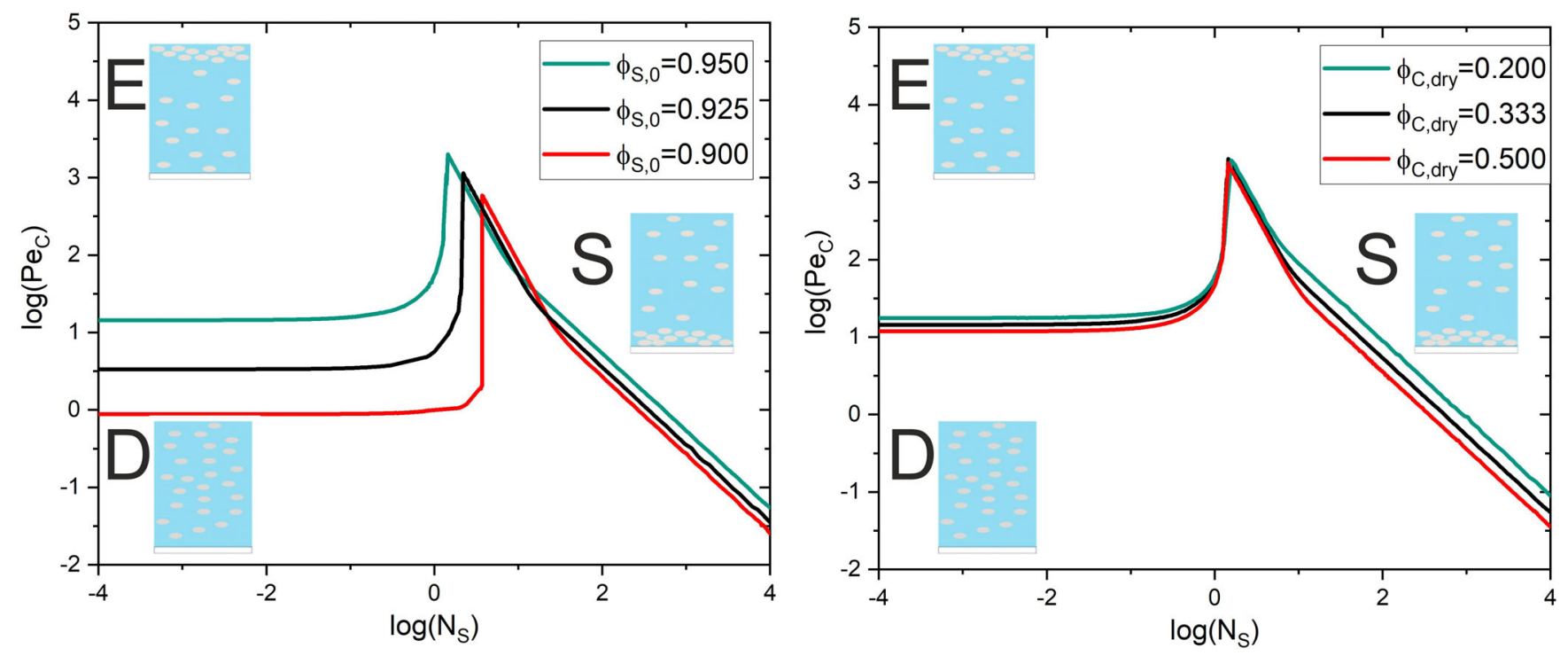

FIGURE 9 Influence of initial solvent and particle volume fraction in the dry film on the drying regime maps at $r_{p}=0.1$. In the left diagram, the borders were drawn using a constant particle volume fraction in the dry film of $\phi_{C, \text { dry }}=0.333$ and the initial solvent volume fraction is modified, showing a reduction of the diffusion regime. This is due to the increase of the particle concentration, making it easier to fulfill the condition of the evaporation regime at lower $P e_{C}$. On the right, the $\phi_{C, \text { dry }}$ is varied with a constant $\phi_{S, 0}=0.95$, which hardly produces any shift of the regime borders due to the low initial concentration $\phi_{C, 0}$

$$
\begin{gathered}
\left.E_{0} \approx U_{C}\right|_{x=h\left(t_{0}\right)}=U_{C, 0} \cdot K\left(\phi_{C, 0}\right), \\
N_{S, \text { crit }} \approx \frac{1}{K\left(\phi_{C, 0}\right)} .
\end{gathered}
$$

The equation predicts that if the sedimentation coefficient $K$ decreases (aspect ratio decreases), $N_{S, \text { crit }}$ will increase at a constant initial particle concentration $\phi_{C, 0}$, leading to an expansion of the evaporation regime. Similar as for $P e_{C, \text { crit }}$, this phenomenon is due to the decrease of mobility at a lower aspect ratio, making it harder for the particles to match the speed of the evaporation front and therefore the region to obtain accumulation at the top of the film is extended to higher sedimentation numbers.

Once the influence of the aspect ratio was determined, it was compared with the influence of the other input parameters, which are not dependent on the physical properties of the material system. In Figure 9, the influence of the initial volume fraction of the solvent $\phi_{S, 0}$ and the influence of the volume fraction of the particles in the dry film $\phi_{C, \text { dry }}$ are displayed. In the right diagram, $\phi_{C \text {,max }}$ was kept constant, while varying $\phi_{S, 0}$. The volume fraction of the polymer is easily determined by adding the volume fractions of all components and equating it to one, knowing the volume fraction of the particles in the absence of a solvent. The reduction of the solvent leads to the obvious conclusion of the expansion of the evaporation regime, due to the greater initial particle concentration, thus reducing the evaporation needed to reach $\phi_{C \text {,max }}$. It must be pointed out that the change of the initial concentration changes the borders almost in the same order of magnitude as changing the aspect ratio. On the contrary, the variation of $\phi_{C, \text { dry }}$ hardly changes the critical dimensionless numbers.
The main reason for the evaporation regime expansion and the low influence of the particle concentration lies in the low mobility of the plate-like particles, which intensifies the effect of the drying driving forces by raising the evaporation rate $E_{0}$, allowing the phase boundary to move faster than the particles can diffuse.

Examples with units on the usage of the drying charts at different aspect ratios can be found in the supporting information. There, it is shown how the change in the drying conditions and particle properties, by means of the $P e_{C}$ and $N_{S}$, can be used to obtain a desired particle distribution in dry film.

\section{5 | SUMMARY AND CONCLUSIONS}

In this article, a simulation model based on past works ${ }^{25,33}$ was developed and automated to obtain new drying regime maps for plate-like particles in water-PVA, utilizing the initial volume fractions of all components and the particle aspect ratio. The input parameters were systematically varied to quantify their influence on the drying regime borders. The component concentration profiles while drying could be calculated with the values for the particle sedimentation, which were obtained experimentally ${ }^{24}$ and from the literature. ${ }^{23,13}$ It was determined that these values could not be reproduced utilizing the analytical expression derived by $\mathrm{He}$ et al. ${ }^{17}$ The decrease of the exponent $K_{2}$ is caused by the change of the particle aspect ratio and therefore the increase of the viscous resistance due to drag. One of the major challenges while simulating the drying regime maps is the scarce experimental data of sedimentation rates of non-spherical particles with narrow particle-size distribution. In future works, polymer particles 
with different aspect ratios will be obtained using synthesis methods with high $r_{p}$ purity found in the literature, ${ }^{34,35}$ which will allow to expand the data base on sedimentation coefficients.

The regime maps were drawn as a function of the aspect ratio and the shifts of the borders were quantified using the critical values of $P e_{C}, N_{S}$, and $P e_{S}$. The decrease of particle mobility resulted in the expansion of the evaporation regime (decrease of $P e_{C, c r i t}$ ) and a small reduction of the sedimentation regime (increase of $N_{S, \text { crit }}$ ). Unfortunately, the physics of the material system limits the range of aspect ratio that could be completely simulated utilizing the method exposed in the present work. This is due to the extremely low values of $K_{2}$.

It could appear that experimentally for the aspect ratios $r_{p}=0.1$ and $r_{p}=0.08$ (see Table 1) the decreasing trend inverses itself, but it must be noted that the values are close to each other and their respective $K_{2} \mathrm{~s}$ are also similar. The overall difference lies under $10 \%$ and a significant change can only be seen at logarithmic scale. Moreover, the reported values with uncertainty intervals overlap themselves, meaning that the reason why $K\left(\phi_{C}, r_{p}=0.1\right)<K\left(\phi_{C}, r_{p}=0.08\right)$ lies in the different methods and experimental deviations while determining $K_{2}$. It is important to mention, that the prediction on the particle concentration relies heavily on how the mobility of the particle is obtained. Furthermore, the influence of the aspect ratio was compared with the influence of the initial solvent volume fraction and the particle volume fraction in the dry film. It was determined that the decrease of solvent volume fraction also expands the evaporation regime, due to the lower mobility of the particles. The increase of $P e_{C, \text { crit }}$ by the increasing $\phi_{S, 0}$ from 0.925 to 0.95 is in the same order of magnitude or equivalent to increasing the aspect ratio $r_{p}$ from 0.10 to 0.15 . This could be later considered while developing a drying process; instead of increasing the initial solvent composition, the aspect ratio of the particles could be changed. Variations of the particle concentration in the dried film did not produce significant changes in the same order of magnitude on the regime map borders.

The simulative results provided in the present manuscript generate new knowledge about the influence of particle geometry while drying particle-polymer composites, highlighting the connection between aspect ratio and particle mobility. These results generate more alternatives while designing drying process strategies, sizing industrial dryers, or formulating coating dispersions.

\section{ACKNOWLEDGMENTS}

The authors acknowledge the financial support from the German Research Foundation (Deutsche Forschungsgemeinschaft), the German Academic Exchange Service (DAAD), and the National Council of Science and Technology (CONACYT) for the research grant provided under no. 471046. The sedimentation rate measurements were done at the Institute of Mechanical Process Engineering and Mechanics (MVM) at KIT. The authors thank Prof. Hermann Nirschl for providing the necessary equipment for the sedimentation rate measurements. Open Access funding enabled and organized by Projekt DEAL.

\section{AUTHOR CONTRIBUTIONS}

Víctor Gracia-Medrano-Bravo: Investigation (equal); writing - original draft (equal). Philip Scharfer: Conceptualization (equal); writing - review and editing (equal). Wilhelm Schabel: Conceptualization (equal); writing - review and editing (equal).

\section{DATA AVAILABILITY STATEMENT}

The data that support the findings of this study are available from the corresponding author upon reasonable request.

\section{ORCID}

Víctor-Alfonso Gracia-Medrano-Bravo (D) https://orcid.org/0000-0002$5462-4623$

\section{REFERENCES}

1. Merklein L, Mink M, Kourkoulos D, et al. Multilayer OLEDs with four slot die-coated layers. J Coat Technol Res. 2019;16(6):1643-1652.

2. Scharfer P, Schabel W, Kind M. Modelling of alcohol and water diffusion in fuel cell membranes-experimental validation by means of in situ Raman spectroscopy. Chem Eng Sci. 2008;63(19):4676-4684.

3. Pandey RP, Shukla G, Manohar M, Shahi VK. Graphene oxide based nanohybrid proton exchange membranes for fuel cell applications: an overview. Adv Colloid Interface Sci. 2017;240:15-30.

4. Kumberg J, Müller M, Diehm R, et al. Drying of lithium-ion battery anodes for use in high-energy cells: influence of electrode thickness on drying time, adhesion, and crack formation. Energ Technol. 2019; 7(11):1900722.

5. Riegel A-L, Borzenkova N, Haas V, Scharfer P, Schabel W. Activity determination of FAD-dependent glucose dehydrogenase immobilized in PEDOT: PSS-PVA composite films for biosensor applications. Eng Life Sci. 2016;16(6):577-585.

6. Cardinal CM, Jung YD, Ahn KH, Francis LF. Drying regime maps for particulate coatings. AIChE J. 2010;56(11):2769-2780.

7. Jeffery GB. The motion of ellipsoidal particles immersed in a viscous fluid. Proc R Soc A. 1922;102(715):161-179.

8. Bertevas E, Fan X, Tanner RI. Simulation of the rheological properties of suspensions of oblate spheroidal particles in a Newtonian fluid. Rheol Acta. 2010;49(1):53-73.

9. Ardekani MN, Costa P, Breugem WP, Brandt L. Numerical study of the sedimentation of spheroidal particles. Int J Multiphase Flow. 2016; 87:16-34.

10. Bird RB, Stewart WE, Lightfoot EN. Transport Phenomena. New York: Wiley; 1960.

11. Perrin F. Mouvement brownien d'un ellipsoide - I. Dispersion diélectrique pour des molécules ellipsoidales. J Phys Radium. 1934; 5(10):497-511.

12. Batchelor GK. Sedimentation in a dilute polydisperse system of interacting spheres. Part 1. General theory. J Fluid Mech. 1982;119: 379-408.

13. Russel WB, Saville DA, Schowalter WR. Colloidal Dispersions (1st Paperback Ed. (with Corr.)). Cambridge: Cambridge University Press; 1991.

14. Dhont JKG. An Introduction to Dynamics of Colloids. 2nd ed. Amsterdam: Elsevier; 2003.

15. Oseen CW. Über die Stokes' sche Formel und Über eine verwandte Aufgabe in der Hydrodynamik. Arkiv för matematik, astronomi och fysik. 1910;6:29.

16. Jansen JW, Kruif CG de, Vrij A. Attractions in sterically stabilized silica dispersions: IV. Sedimentation. J Colloid Interface Sci 1986;114(2): 501-504.

17. He $P$, Mejia $A F$, Cheng $Z$, et al. Hindrance function for sedimentation and creaming of colloidal disks. Phys Rev E Stat Nonlin Soft Matter Phys. 2010;81(2 Pt 2):26310.

18. Weeks JD, Chandler D, Andersen HC. Role of repulsive forces in determining the equilibrium structure of simple liquids. J Chem Phys. 1971;54(12):5237-5247. 
19. Chaikin PM, Donev A, Man W, Stillinger FH, Torquato S. Some observations on the random packing of hard ellipsoids. Ind Eng Chem Res. 2006;45(21):6960-6965.

20. Buscall R, Goodwin JW, Ottewill RH, Tadros TF. The settling of particles through Newtonian and non-Newtonian media. J Colloid Interface Sci. 1982;85(1):78-86.

21. Cheng PY, Schachman HK. Studies on the validity of the Einstein viscosity law and Stokes' law of sedimentation. J Polym Sci. 1955;16(81): 19-30.

22. de Kruif CG, Jansen JW, Vrij A. A Sterically Stabilized Silica Colloid as a Model Supramolecular Fluid. In Physics of Complex and Supramolecular Fluids (eds S.A. Safran and N.A. Clark). New York: Wiley-Interscience; 1987:315-346.

23. van der Kooij FM, Philipse AP, Dhont JKG. Sedimentation and diffusion in suspensions of sterically stabilized colloidal platelets. Langmuir. 2000;16(12):5317-5323.

24. Gracia-Medrano-Bravo V-A, Gröne J, Baesch S, Scharfer P, Schabel W. Influence of particle shape on the drying regime maps for platelike particle-polymer composites. Langmuir. 2020;36(22):62456253.

25. Baesch S, Scharfer P, Schabel W, Francis L. Influence of the drying conditions on the particle distribution in particle-filled polymer films: predictive simulation of the particle distribution during drying. J Compos Mater. 2017;51(24):3391-3403.

26. Routh AF, Zimmerman WB. Distribution of particles during solvent evaporation from films. Chem Eng Sci. 2004;59(14):2961-2968.

27. Jeck S, Scharfer P, Schabel W, Kind M. Water sorption in poly(vinyl alcohol) membranes: an experimental and numerical study of solvent diffusion in a crosslinked polymer. Chem Eng Process Process Intensif. 2011;50(5-6):543-550.

28. Schlünder E-U. Einführung in Die Stoffübertragung. Stuttgart: Thieme; 1984.

29. Stephan P. VDI-Wärmeatlas (11., bearb. und erw. Aufl.). Berlin: Springer Vieweg; 2013.
30. Schabel W. Inverse Mikro-Raman-Spektroskopie - Eine neue Messmethode zur Untersuchung lokaler Stofftransportvorgänge in dünnen Filmen, Folien und Membranen. Chemie Ingenieur Technik. 2005;77(12):1915-1926.

31. Kachel S, Scharfer P, Schabel W. Measurements and predictive modeling of water diffusion coefficients in bovine serum albumin/polymer blends for biosensors. J Appl Polym Sci. 2017;134(40):45368.

32. Merklein L, Eser JC, Börnhorst T, Könnecke N, Scharfer P, Schabel W. Different dominating mass transport mechanisms for drying and sorption of toluene-PMMA films - visualized with Raman spectroscopy. Polymer. 2021;222:123640.

33. Baesch S, Price K, Scharfer P, Francis L, Schabel W. Influence of the drying conditions on the particle distribution in particle filled polymer films: experimental validation of predictive drying regime maps. Chem Eng Process Process Intensif. 2018;123:138-147.

34. Ho CC, Keller A, Odell JA, Ottewill RH. Preparation of monodisperse ellipsoidal polystyrene particles. Colloid Polym Sci. 1993;271(5):469-479.

35. Ahn SJ, Ahn KH, Lee SJ. Film squeezing process for generating oblate spheroidal particles with high yield and uniform sizes. Colloid Polym Sci. 2016;294(5):859-867.

\section{SUPPORTING INFORMATION}

Additional supporting information may be found in the online version of the article at the publisher's website.

How to cite this article: Gracia-Medrano-Bravo V-A, Scharfer P, Schabel W. On the drying kinetics of non-spherical particle-filled polymer films: A numerical study. AIChE J. 2021; e17398. doi:10.1002/aic.17398 